\title{
Non-Extensive Statistical Mechanics - a Possible Basis for Modelling Processes in Computer Memory System
}

\author{
D. STRZAŁKA* \\ Rzeszów University of Technology, Department of Distributed Systems, W. Pola 2, 35-959 Rzeszów, Poland \\ Tsallis definition of non-extensive entropy introduced in 1988 has been considered to obtain new possibilities \\ to construct generalized thermodynamical basis for statistical physics expanding classical Boltzmann-Gibbs \\ thermodynamics for nonequilibrium states. During the last two decades this $q$-generalized theory has been \\ successfully applied to considerable number of physically interesting complex phenomena. In the paper the \\ possible thermodynamical basis of processes will be presented in nowadays computer systems in the case of \\ memory behaviour.
}

PACS numbers: 89.20.Ff, 05.20.-y, 05.90.+m

\section{Introduction}

As it is known Boltzmann-Gibbs (BG) entropy $S$, which is a base of many considerations, for $W$ equiprobable microstates is based on one of the most important equations

$$
S_{\mathrm{BG}}=k \ln W,
$$

while Tsallis definition of $S_{q}$ non-extensive entropy gives its $q$-generalization, by $[1,2]$

$$
S_{q}=k \ln _{q} W,
$$

where

$$
\ln _{q}(W)=\frac{W^{1-q}-1}{1-q}, \quad W>0 \quad \text { and } \quad q \in R .
$$

Tsallis definition of entropy assumes that this is a non-additive system property and the degree of non-extensivity can be expressed by the $q$ parameter [2]. It is assumed that the BG entropy can be used everywhere where the system is in equilibrium state or close to it (quasi-equilibrium). This means that from a thermodynamical point of view the entropy production is at the lowest possible level (the Onsanger relations) [3].

Tsallis entropy considered as a possible generalization of BG entropy has many interesting properties, but especially the idea of non-extensivity is the most interesting one. It leads to many interesting and fierce discussions also including a strong criticism [4-7]. But analyzing many papers (see the references in [8]) one can see that the non-extensive entropy helps to find a very elegant basis for many of those problems, which haven't got it so far. Such a case is the problem of possible thermodynamical explanation of Lévy distributions [9]. It is worth noticing that starting form the problem of entropy $S$ maximaliza-

\footnotetext{
* e-mail: strzalka@prz.edu.pl
}

tion under given probability distribution $p(x)$ in the case of Boltzmann-Gibbs entropy one will have

$$
S_{\mathrm{BG}}[p(x)]=-k_{\mathrm{B}} \int p(x) \ln [\sigma p(x)] \mathrm{d} x,
$$

with the solution given by Gaussian distribution [9]

$$
p(x)=\frac{1}{\sqrt{2 \pi} \sigma} \mathrm{e}^{-\frac{x^{2}}{2 \sigma^{2}}} .
$$

For Tsallis entropy similarly it will be

$$
S_{q}[p(x)]=-k \frac{1-\int[\sigma p(x)]^{q} \frac{d x}{\sigma}}{q-1}, \quad q \in R,
$$

with the solution for $q<3$ given by $[9,12]$

$$
p(x)=N_{q} e_{q}^{-B_{q}\left(x-\bar{\mu}_{q}\right)^{2}}
$$

where

$$
\begin{aligned}
& N_{q}=\left\{\begin{array}{lcc}
\frac{\Gamma\left[\frac{5-3 q}{2-2 q}\right]}{\Gamma\left[\frac{2-q}{1-q}\right]} \sqrt{\frac{1-q}{\pi} B_{q}} & \text { for } & q<1, \\
\frac{\Gamma\left(\frac{1}{q-1}\right)}{\Gamma\left(\frac{3-q}{2(q-1)}\right) \sqrt{\frac{\pi}{(q-1) B_{q}}}} & \text { for } & 1<q<3,
\end{array}\right. \\
& \bar{\mu}_{q}=\int x \frac{[p(x)]^{q}}{\int[p(x)]^{q} \mathrm{~d} x} \mathrm{~d} x \equiv\langle x\rangle_{q}, \\
& B_{q}=\left[(3-1) \bar{\sigma}_{q}^{2}\right]^{-1}
\end{aligned}
$$

and $e_{q}^{x}$ denotes the $q$-exponential function defined as $[1,2]$

$$
e_{q}^{x}=[1+(1-q) x]^{\frac{1}{1-q}} .
$$

When $|x| \rightarrow \infty$ Eq. (7) behaves like

$$
p(x) \approx x^{\frac{-2}{q-1}} \text {. }
$$

Tsallis showed $[9,10]$ that such probability distributions correspond with Lévy $\alpha$-stable distributions $(\alpha \in\langle 0,2\rangle)$ where 


$$
\alpha=\frac{3-q}{q-1} \quad \text { for } \quad \frac{5}{3}<q<3 .
$$

In statistics there are a few posibilites for calculations of the $\alpha$ parameter, for example [11]: Hill estimator, maximum likelihood estimator or (the simplest but not necessary good) graphical method where the right tail of probability distribution (or density function, i.e., $1-F(x)$ ) is plotted on a log-log scale giving almost a stright line with some slope, which can be calculated by the last mean squares method and $\alpha=|\gamma+1|$ where $\gamma=$ slope. In the presented paper there was taken the approach that was proposed in [12] where one can find an analitical method for calculations of cumulated heavy tailed distributions with their explanation based on Tsallis entropy. This approach should be taken in order to attain a better stability of analysis.

The cumulative form of the distribution given by Eq. (7), namely

$$
P_{ \pm}(x)=\mp \int_{ \pm \infty}^{x} p\left(x^{\prime}\right) \mathrm{d} x^{\prime},
$$

where in Eq. (11) the + and - signs correspond to the right and left wings of the distribution, can be given as

$$
\begin{gathered}
P_{ \pm}(x)=N_{q}\left(\frac{\sqrt{\pi} \Gamma\left(\frac{1}{2}(3-q) \beta\right)}{2 \Gamma(\beta) \sqrt{\frac{B_{q}}{\beta}}}\right. \\
\left. \pm\left(x-\bar{\mu}_{q}\right)_{2} F_{1}(\alpha, \beta ; \gamma ; \delta)\right),
\end{gathered}
$$

where $\alpha=\frac{1}{2}, \beta=\frac{1}{q-1}, \gamma=\frac{3}{2}, \delta=-B_{q}(q-1)\left(\bar{\mu}_{q}-x\right)^{2}$ and ${ }_{2} F_{1}(\alpha, \beta ; \gamma ; \delta)$ is the Gauss hypergeometric function.

The maximalization of $S_{q}$ entropy given by (6) leads us to the possible explanation of power-laws existence (see Fig. 1). They are usually connected with fractals [13] and non-equilibrium behaviour of many systems. The existence of such distributions is well known and it was observed in many natural systems (see for example [2]). Basing on P. Lévy approach $[14,15]$ we can relate some aspects of power-laws to Lévy processes as very important ones in dynamics modelling because they can be used everywhere when the problem of infinite variance appears. The graphical visualizations of Lévy processes are characterized by the existence of pikes, which in Gaussian processes don't exist [11]. These distributions were rather considered as the "accidents during normal work" [14], but thanks to Tsallis work [9] it can be considered that they can have the thermodynamic basis and represent such cases when the non-equilibrium appears.

Here, it should be emphasized that almost all of the analysis of computer systems so far done were based on the assumptions that they are rather closer to the BG entropy than to the Tsallis approach. As an example, the case of computer networks can be shown where modeling of traffic behaviour is based on model with finite variance (Gaussian random noise or Brownian motion expanded on fractal models) [16] or the better example - queuing theory models with operational approach where the analysis is based on the assumption that the modeling

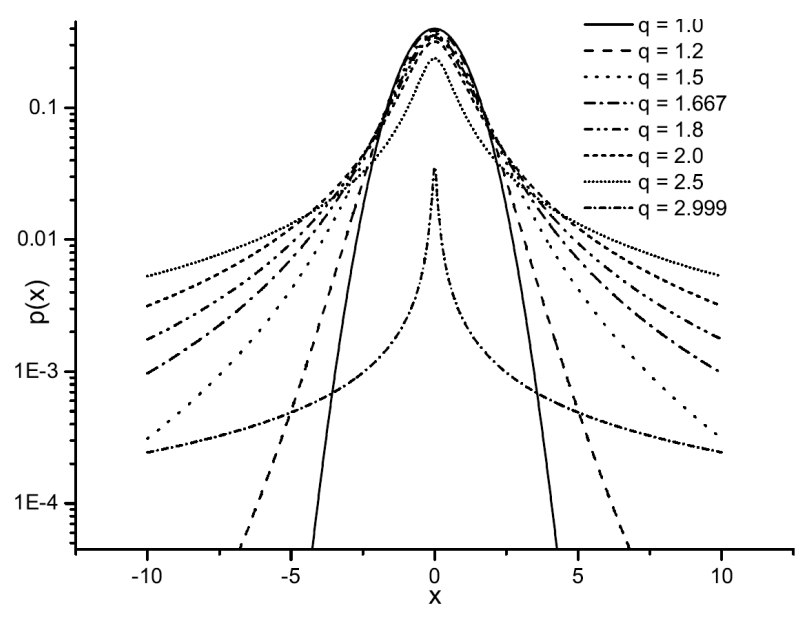

Fig. 1. A family of distributions given by Eq. (7) presented on a log-lin scale. When $q>5 / 3$ the slowly decaing tails ("heavy tails") are well visible.

is possible when the queuing system is in equilibrium state [17]. The reason for this is very simple: in computer engineering there is a lack of knowledge based on a Tsallis entropy. This is very incomprehensible because almost everyone agrees that the nowadays computer systems work under conditions that cause their dynamical behaviour as an intrinsic feature, but surprisingly almost no one takes into account this problem during the research. Probably Tsallis idea isn't appreciated enough or in some cases the computer engineering is still considered rather as a mathematical theory not as a physical science [18]. Meanwhile, for better understanding of processes that appear inside computer systems the proper thermodynamical basis is needed. It seems that such a basis can be found in Tsallis proposal.

\section{Computer memory system}

The system notion, in the wide range of disciplines from ecology to physics, social sciences and informatics, has received a significant attention in the last years. Generally, the system can be interpreted as a structure consisting of the (not necessary large) number of interdependent elements [21]. In order to understand the behaviour of any system there are two possible approaches that can be characterized by two notions: reductionism and the opposite holism. The reductionistic approach is proper for the simple (or complicated) systems [22, 23], while each complex system should be analyzed not only by knowledge of behaviour of its individual elements but also how they act together [24]. Such an approach should be also used in the case of computer systems because they are no longer simple (complicated) systems but they are rather the complex ones $[21,25,27]$ working in both: algorithmic and interactive mode [18, 19] and during processing are governed by the long-range dependencies $[21,26,28,29]$. This means that some kind of paradigm change is needed in the approach for computer systems 
analysis. But such a new approach needs an appriopriate definition of thermodynamical basis because each real computer system is a physical not mathematical system, so it needs energy for its activity and that's why the problem of entropy production appears.

\subsection{Memory subsystem}

The computer systems consist of many interdependent subsystems. The dependencies existing between them are very complex. Moreover, it should also be noticed that first, the dependencies exist inside these subsystems [30] and second, the structure of subsystems in many cases is hierarchical [31]. An example of such a subsystem is computer memory. Its hierarchical structure is a natural consequence of physical (capacity, access time) and economical (cost per unit) conditions (see Fig. 2) that govern it.

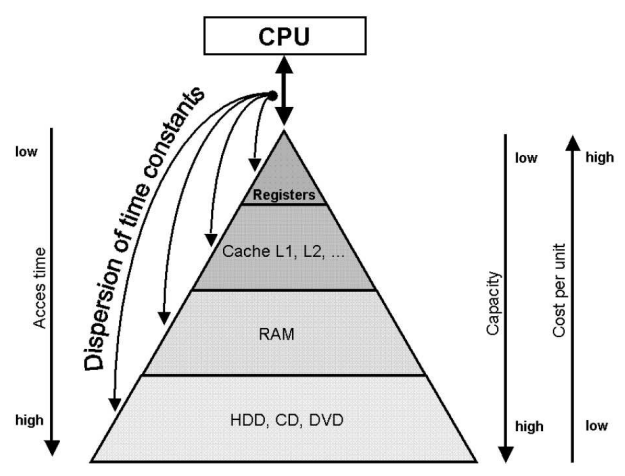

Fig. 2. Hierarchical structure of memory subsystem.

There are a few interesting properties of hierarchical structure of memory subsystem but the most important one is a time constants dispersion that exists inside. To explain this problem, let's assume that the Central Processing Unit (CPU) has $1 \mathrm{GHz}$ clock frequency, so each clock tact lasts for 1 ns. A processing is possible when CPU has an access for data. In Turing machine model memory is built of infinite tape [32] and the access time to each memory 'cell' is equal 0 [33]. In reality the situation is different because during processing there is a need to take data from different levels of implemented memory: starting from registers and finishing even on the Interent level. For each level one will have different values of delays. A time delay for registers is $<3 \ldots 4$ tacts (access time $<3-4$ ns, i.e. comparable order of magnitude), for caches $L 1, L 2$. . about $20-30$ tacts (access time $20-30 \mathrm{~ns}$, i.e. $\approx 1$ order of magnitude), for RAM 60-70 tacts (access time $\approx 70$ ns, i.e. $\approx 2$ orders of magnitude), while for $\mathrm{HD}$ it is $20-30 \mathrm{~ms}$ that is $20-30 \times 10^{6}$ tacts or in other words $\approx 7$ orders of magnitude. For other levels of memory subsystem (CD, DVD, USB Memory, etc.) this dispersion is even bigger. The existence of time constants dispersion and solutions used for management of access to the memory system (for example a mechanism of virtual memory [20]) suggest that the behaviour of the whole system should be very dynamical. If it is the truth, there is a need to have a proper thermodynamical basis that can help modeling behaviour of this subsystem.

\subsection{Perfmon - Windows system monitor}

It should be also noticed, that the whole problem is more complicated than it seems to be because the behaviour of memory (sub)system is also depended on or (the better description) governed by operating system, which has implemented mechanisms of memory management. Moreover, the complex processes inside memory and its dynamical behaviour can depend also on the character of input data and realized tasks [21]. It also seems that there isn't any way to trace memory behaviour without the influence on its behaviour, because each monitor (computer program) that will trace the actual state of memory will be processed inside the computer system and "disturb" the results of research. However, there is a possibility to do this and surprisingly is given by operating system. This solution can be achieved by mechanism implemented in operating system, because it itself needs information of actual state of computer subsystems for its normal work. For example, such mechanisms are implemented in Windows ${ }^{\mathrm{TM}}$ family of operating systems and are a part of administration tools. The access is given from an administrator level by perfmon tool and this solution was taken to present the main results of this work.

Perfmon allows tracking the whole system by graphical visualizations or data records for further statistical analysis. The smallest time interval for data recording can be set up to $1 \mathrm{~s}$. and it seems that this resolution is enough, because during $1 \mathrm{~h}$ it gives 3600 observations. Obviously it means that if one has got a CPU clock with $f=1 \mathrm{GHz}$ the resolution $1 \mathrm{~s}$. can result a lost of many interesting system properties, but on the other hand the assumed dispersion in memory (sub)system can achieve 20-30 (or more) ms, so it means that this resolution is a sensible compromise. Because perfmon is a normal computer program it is also processed by CPU, but it shows data from system counters that are normally traced by operating system thus its influence on computer system performance is minimal (less than 1\%) and is mostly generated by visualizations of traced data or by the necessity of data recording on disks. The recorded data can be exported to the statistical packages.

\section{Results of investigations}

The investigations presented in this paper are the results of almost $42 \mathrm{~h}$ of tracking of two chosen counters:

- Memory $\backslash$ Pages Faults/s, which shows how many times information being requested is not where the application expects it to be. The information must either be retrieved from another location in memory or from the pagefile, i.e., the memory system,

- Memory $\backslash$ Cache Faults/s, which shows how often data sought in the File System Cache is not found there. This value should be as small as possible. 
These two counters were taken into account because they can help relate the fault rate of the cache to the fault rate in the system as a whole. Memory $\backslash$ Cache Faults/s is a component of Memory $\backslash$ Page Faults/s and their ratio indicates the proportion of faults occurring in the cache. A high rate of cache faults can indicate a memory shortage. But it can also indicate that the organization of data on disk is inefficient. However, we wouldn't analyze the proportion given by one value (e.g. its average), but some properties of probability density functions for above mentioned counters given by time series recorded in time interval with the resolution $1 \mathrm{~s}$. This approach will show the possible thermodynamical basis of these processes and will help establish their (possible) degree of non-extensivity. The need to have the right thermodynamical basis is crucial for further investigations especially if one wants to understand all the phenomena that appear and govern the computer systems and their subsystems.

The research was done on Dell Inspiron 6400 computer with Intel Core Duo (T2540) $2 \mathrm{GHz}$ processor with 2 GB of RAM, 160 GB HD (5400 rpm) under Windows $\mathrm{XP}^{\circledR}$ Professional + SP2 (compilation 2600). As it can be seen during the experiment there weren't any special scenarios, benchmarks or special testing programs used. This approach excludes the possibility of being repeated but it was done because normally during work with the computer we don't use any special hard workload - we mostly use the computers for office processing or entertainment. Such a workload can be considered as a 'normal average' work and it better reflects the real nature of tasks processed in computer systems. During a normal work there were used:

- 2 www explorators $\left(\right.$ Opera ${ }^{\circledR}+$ Firefox $\left.^{\circledR}\right)$,

- Webdesign ${ }^{\circledR}$ (apache + php + mysql server $)$,

- MS Office ${ }^{\circledR}$ (Word + Excel),

- Windows Media Player ${ }^{\circledR}$ (projection of 2 movies),

- Winamp ${ }^{\circledR}$,

- internet communicator,

- Foxmail $^{\circledR}$ (mail program that after every 10 minutes checked mail).

There were collected $150 \times 10^{3}$ observations $(\approx 42 \mathrm{~h}$ of data recording) and the recorded data represents the memory cache faults (denoted by $X(t)$ ) and the total number of memory page faults (denoted by $Y(t)$ ). Basing on Fig. 3 and 4 one can see the visualisations of these two time series where the successive values are divided by calculated standard deviations.

$>$ From Figs. 3 and 4 one can see the dynamical nature of both processes; this indicates that during normal work of computer system appear such phenomena that

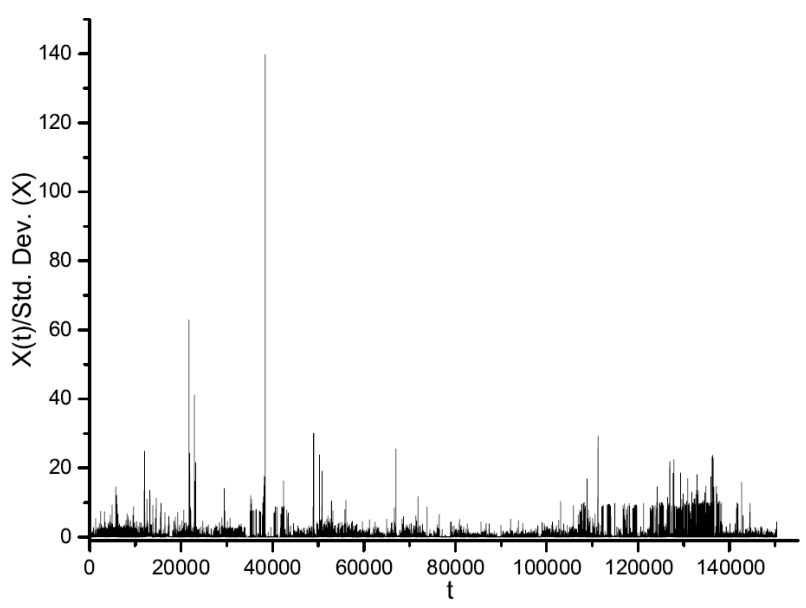

Fig. 3. Time series that represents the process of cache faults $X(t)$ divided by the value of its standard deviation.

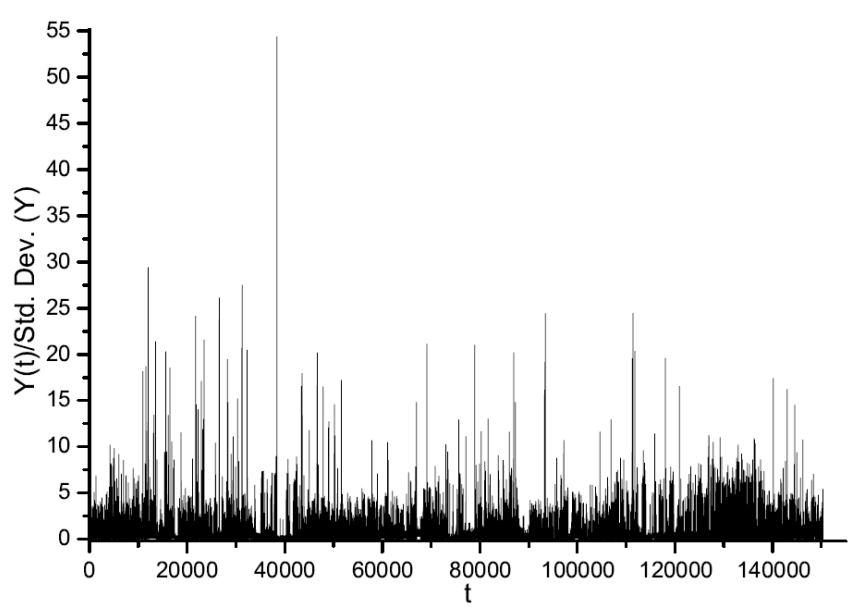

Fig. 4. Time series that represents the process of number of pages faults $Y(t)$ divided by the value of its standard deviation.

force the implemented mechanisms of memory management (memory subsystem) to be in highly extreme states. This problem will be better visible when the graphical visualisations of distributions of increments for analyzed processes will be given. In the case of cache increments and pages increments as well the distributions plotted on a log-lin scale (see Figs. 5 and 6 ) show that their tails vanish slowly than fitted, by estimated values of processes mean and varaince, the Gaussian ones. These distributions indicate the dynamical behaviour of analyzed system and show that the probability of appearance of extreme increments can be governed by "heavy tailed" distributions. Because Gaussian processes are connected by the Eqs. (4) and (5) with Boltzmann-Gibbs entropy and the tails of analyzed processes vanish slowly, Tsallis definition of entropy (3) with the possible distributions given by (7) seems to be the right one and for these processes a modeling based on Lévy $\alpha$-stable processes can 
be used.

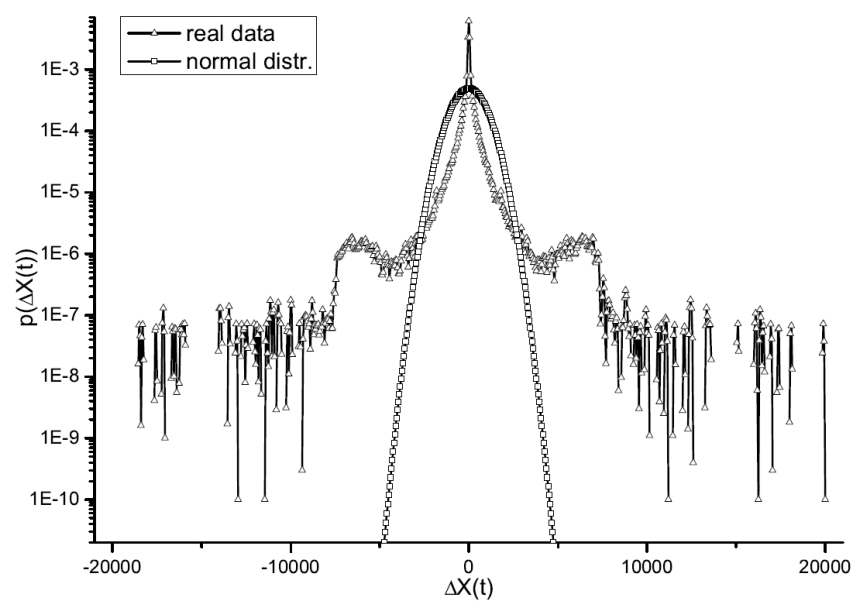

Fig. 5. The distribution of cache faults increments $\Delta X(t)=X(t+1)-X(t)$ plotted on a log-lin scale. The slowly decaying tails of real distribution obtained form kernel estimator in comparison to the Gaussian distribution (line with dots) are visible.

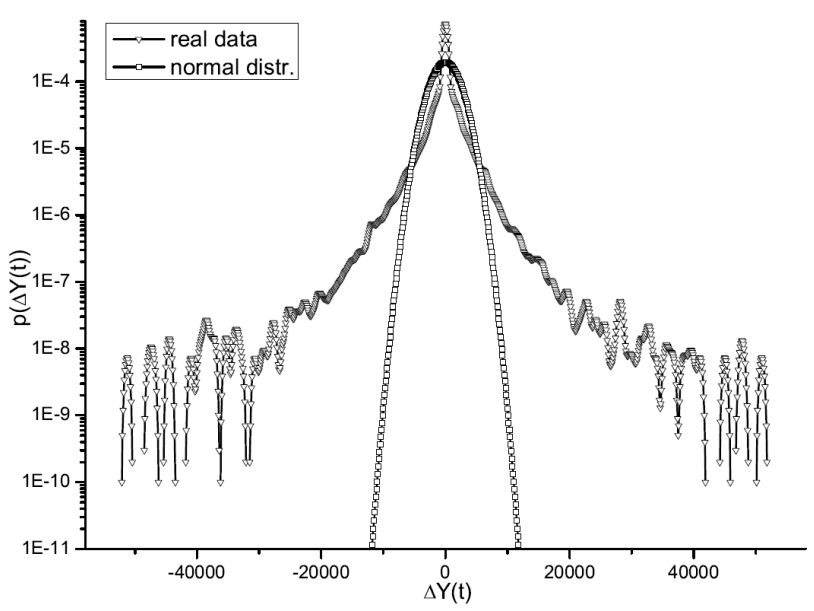

Fig. 6. The distribution of pages faults increments $\Delta Y(t)=Y(t+1)-Y(t)$ plotted on a log-lin scale. The slowly decaying tails of real distribution obtained from kernel estimator in comparison to the Gaussian distribution (line with dots) are visible.

Basing on the approach given in [12] there were estimated the values of $q$ parameter for analyzed time series. From the Eq. (9) it can be seen that the behaviour of tail for large $x$ is given by power-law $-2 /(q-1)$. But remembering that in used approach we consider the cumulative distributions we are forced to take this into account one must subtract 1 during the estimations of slope and finally the degree of non-extensivity expressed by $q$ parameter can be obtained from $q=2 /(\alpha+1)+1$. In the case of cache faults the estimated value of $q=1.58$ while for pages faults it is $q=1.44$ (see Figs. 7 and 8 for the details). The obtained results indicate that the

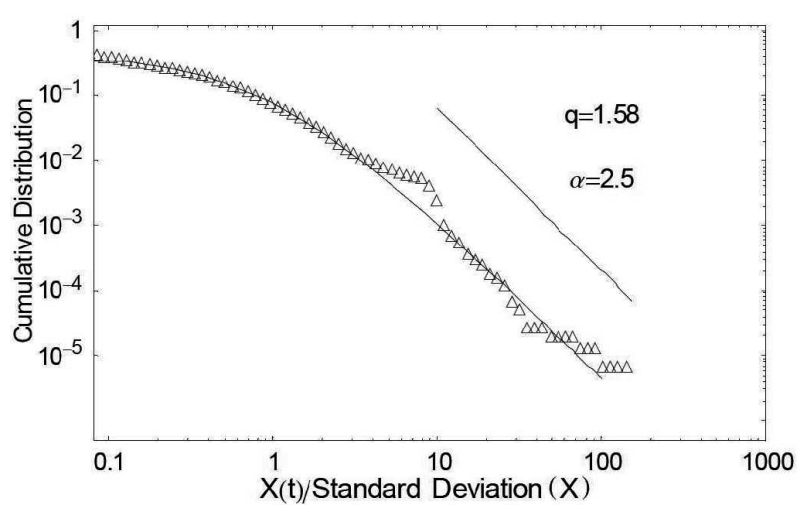

Fig. 7. The cumulative probability distribution of cache faults plotted on a log-log scale (triangles). The slope of estimated $q$-Gaussian cumulative distribution $\alpha=2.5$; taking into account Eq. (9) and remembering that there was analyzed the cumulative distrbution one can obtain $q=2 /(\alpha+1)+1$ and for analyzed case it's $q=1.58$. The continues line shows $q$-Gaussian distribution (given by (12)) with $q=1.58$.

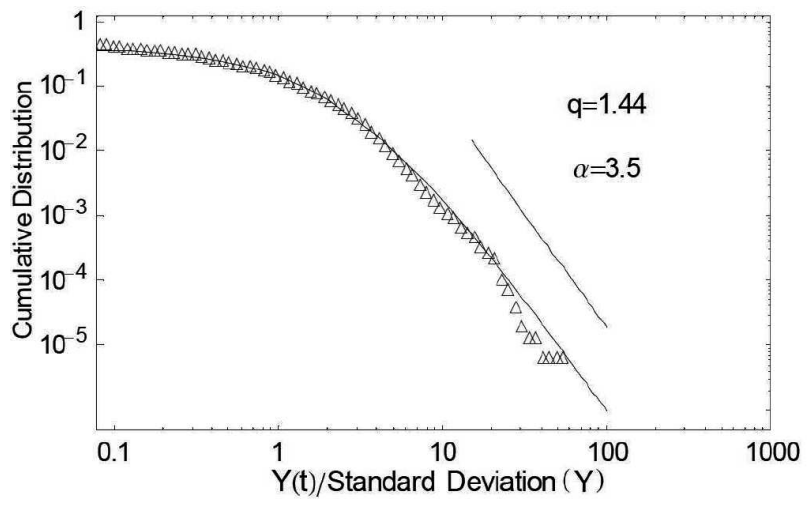

Fig. 8. The cumulative probability distribution of pages faults plotted on a log-log scale (triangles). The slope of estimated $q$-Gaussian cumulative distribution $\alpha=3.5$; taking into account Eq. (9) and remembering that there was analyzed the cumulative distrbution one can obtain $q=2 /(\alpha+1)+1$ and for analyzed case it's $q=1.44$. The continues line shows $q$-Gaussian distribution (given by (12)) with $q=1.44$.

nonextensive statistical mechanics based on the generalized Tsallis entropy seems to offer a very interesting theoretical framework for calculations of the probability distributions for analyzed processes. Obviously these results need further studies, but it can be assumed that data obtained from computer memory counters used by operating system for assurance of its appropriate performance can be seen in terms of the nonextensivity. The existence of such phenomena provides further arguments for considerations that the nonextensive statistical mechanics can be an inspiring framework for quantifying the computer systems dynamics. 


\section{Conclusions}

In the presented paper it has been shown that in the case of modelling of computer memory system behaviour Tsallis non-extensive entropy as a thermodynamical basis can be used. The memory subsystem has the hierarchical structure and the dispersion of time constants that can reach a few orders of magnitude, leads to the existence of very dynamic phenomena that should have a proper thermodynamical basis. This is very important especially if one realizes that computer systems are the complex ones and a paradigm change for their analysis should be done. Despite that the research was done during normal use of computer (there weren't used any special tests or benchmarks), the presented results showed that the computer systems normally work under conditions that are far from equilibrium state. The presented results can be used for further investigations especially for the analysis of the existence of long-range dependent processes in computer systems.

\section{References}

[1] C. Tsallis, J. Stat. Phys. 52, 479 (1988).

[2] E.M.F. Curado, C. Tsallis, J. Phys. A, Math. Gen. 24, L69 (1991).

[3] I. Prigogine, I. Stengers, Order out of Chaos. Man's new dialogue with nature, Bantam Books, New York 1984.

[4] P. Grassberger, Phys. Rev. Lett. 95, 140601 (2005).

[5] C. Tsallis, arXiv:cond-mat/0511213v1.

[6] M. Nauenberg, Phys. Rev. E 67, 036114 (2003).

[7] C. Tsallis, Phys. Rev. E 69, 038101 (2004).

[8] C. Tsallis, http://tsallis.cat.cbpf.br/ TEMUCO.pdf , (2009).

[9] C. Tsallis, S.V.F. Levy, A.M.C. Souza, R. Mayanard, Phys. Rev. Lett. 75, 3589 (1995).

[10] D. Prato, C. Tsallis, Phys. Rev. E 60, 2398 (1999).

[11] R. Weron, Int. J. Mod. Phys. C 12(2), 209 (2001).

[12] R. Rak, S. Drożdż, J. Kwapień, Physica A 374, 315 (2007).

[13] M.E.J. Newman, Contemporary Physics 46, 323 (2005).

[14] R.N. Mantegna, H.E. Stanley, An Introduction to Econophysics: Correlations and Complexity in Finance, Cambridge University Press, Cambridge 1999.
[15] J.P. Nolan, Stable Distributions - Models for Heavy Tailed Data, Birkhauser, Boston 2009 (Unfinished manuscript, Chapter 1 online at academic2. american. edu/ jpnolan ).

[16] M.S. Taqqu, V. Teverovsky, W. Willinger, Fractals 5 , 63 (1997).

[17] E.D. Lazowska, J. Zahorjan, G.S. Graham, K.C. Sevcik, Quantitative System Performance, Prentice Hall, New Jersey 1984.

[18] P. Wegner, D. Goldin, Comm. of the ACM 46, 100 (2003).

[19] P. Wegner, Comm. of the ACM 40, 80 (1997).

[20] A. Silberschatz, P.B. Galvin, G. Gagne, Operating System Concepts, John Wiley \& Sons, Inc., New York 2004.

[21] F. Grabowski, D. Strzałka, Fundam. Inform. 72, 155 (2006).

[22] L.A.N. Amaral, J.M. Ottino, Chem. Eng. Sci. 59, 1653 (2004).

[23] D. Strzałka, F. Grabowski, in: Proc. 2008 Conf. On Human System Interactions, Vol. 1-2, Kraków 570 (2008).

[24] L. von Bertalanffy, General System theory: Foundations, Development, Applications, George Braziller, New York 1976.

[25] F. Grabowski, D. Strzałka, in: New technologies in computer networks, Eds. B. Pochopień, A. Kwiecień, A. Grzywak, J. Klamka, WKiŁ, Warsaw 109 (2006).

[26] D. Strzałka, F. Grabowski, Int. J. Mod. Phys. C 19 , 1443 (2008).

[27] D. Strzałka, F. Grabowski, in: Computer Networks New Technologies, Eds. B. Pochopień, A. Kwiecień, A. Grzywak, J. Klamka, WNT, Gliwice 133 (2005).

[28] F. Grabowski, D. Strzałka, in: Proc. of the CSEP 2005, Warsaw University Press 168 (2005).

[29] F. Grabowski, D. Strzałka, in: Proc. of the CS\&P 2007, Warsaw University Press 235 (2007).

[30] D. Strzałka, F. Grabowski, Fundam. Inform. 85, 455 (2008).

[31] K. Hwang, Advanced Computer Architecture: Parallelism, Scalability, Programmability, McGraw-Hill Book Co., New York 1993.

[32] R. Penrose, The Emperor's New Mind: Concerning Computers, Minds, and The Laws of Physics, Oxford Univ. Press, Oxford 1989.

[33] L.A.N. Amaral, J.M. Ottino, Eur. Phys. J. 38, 47 (2004). 